\title{
Convergence and completeness for square-well Stark resonant state expansions
}

Cite as: J. Math. Phys. 59, 113501 (2018); https://doi.org/10.1063/1.5042523

Submitted: 01 June 2018 . Accepted: 16 October 2018 . Published Online: 02 November 2018

David Juhasz, Miro Kolesik, and Per Kristen Jakobsen

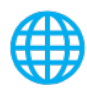

View Online
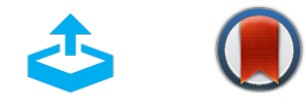

Export Citation

CrossMark

\section{ARTICLES YOU MAY BE INTERESTED IN}

Constructing a partially transparent computational boundary for UPPE using leaky modes

Journal of Mathematical Physics 60, 083505 (2019); https://

doi.org/10.1063/1.5099193

Leaky-mode expansion of the electromagnetic field inside dispersive spherical cavity

Journal of Mathematical Physics 59, 033501 (2018); https://

doi.org/10.1063/1.5006956

Electromagnetic radiation and the self-field of a spherical dipole oscillator

American Journal of Physics 88, 693 (2020); https://doi.org/10.1119/10.0001348

\section{Sign up for Alerts}

Journal of

Mathematical Physics

Receive the latest research updates 


\title{
Convergence and completeness for square-well Stark resonant state expansions
}

\author{
David Juhasz, ${ }^{1}$ Miro Kolesik, ${ }^{2}$ and Per Kristen Jakobsen ${ }^{1}$ \\ ${ }^{1}$ Department of Mathematics and Statistics, The Arctic University of Norway, \\ 9019 Troms $\phi$, Norway \\ ${ }^{2}$ College of Optical Sciences, University of Arizona, 1630 East University Boulevard, \\ Tucson, Arizona 85721, USA
}

(Received 1 June 2018; accepted 16 October 2018; published online 2 November 2018)

\begin{abstract}
In this paper, we investigate the completeness of the Stark resonant states for a particle in a square-well potential. We find that the resonant state expansions for target functions converge inside the potential well and that the existence of this convergence does not depend on the depth of the potential well, $V_{0}$. By analyzing the asymptotic form of the terms in these expansions, we prove some results on the relation between smoothness of target functions and the asymptotic rate of convergence of the corresponding resonant state expansion and show that the asymptotic rate of convergence is also independent of $V_{0}$, but the absolute size terms in the series asymptotically goes as $V_{0}^{-1}$. Published by AIP Publishing. https://doi.org/10.1063/1.5042523
\end{abstract}

\section{INTRODUCTION}

Decaying quantum states were first introduced in 1928 by Gamow ${ }^{1,2}$ and independently by Gurney and Condon ${ }^{3,4}$ in the context of nuclear physics, to describe long-lived wave functions of particles that eventually "escape" from a confining but unstable potential. This was however not the first time decaying eigenstates were used in physics. As early as 1884 , Thomson ${ }^{5}$ used them to describe decay phenomena in electromagnetism. Characterization of the unstable states using the absence of incoming waves was first introduced by Siegert ${ }^{6}$ in the context of the nuclear scattering matrix. The Siegert characterization was taken up by Peierls, ${ }^{7}$ Couteur, ${ }^{8}$ and Humblet ${ }^{9}$ and developed into a powerful tool in nuclear scattering theory. The wave functions satisfying the Siegert outgoingwave conditions became known as resonant states and their properties have been investigated for many years (for example, Refs. 10-19).

The fact that resonant states decay exponentially in time implies that it is more likely to find the released particle far from the nucleus than closer to it since it is more likely to have been released by an earlier time than a later one. The resonant states are thus not normalizable, at least not in the standard sense introduced by von Neumann when he gave a proper mathematical foundation ${ }^{20}$ for a subset of the Dirac formalization of quantum mechanics ${ }^{21}$ in terms of Hilbert spaces for the states and Hermitian operators for the observables. Subsequently, after many intervening years of steady mathematical progress involving many people, an extended mathematical foundation for the full Dirac formalization, involving rigged Hilbert spaces, was developed by Gelfand. ${ }^{22}$ It turned out that the context of rigged Hilbert spaces was flexible enough to also include resonant states even if such states were not part of Dirac's original formalization of quantum mechanics. However, the theory of rigged Hilbert spaces is perhaps a bit heavy on the mathematical side, and therefore, several other extensions of the von Neumann foundation of quantum mechanics designed to accommodate resonant states has been developed over the years. ${ }^{23-28}$ Over and above these mathematical developments, a key issue involving resonant states has been their physical interpretation. The fact that they cannot be normalized in the Hilbert space setting means that the Born interpretation of quantum states in terms of probability theory fails. However, it has been shown that a state with infinite norm can be given a probabilistic interpretation by considering an expanding sphere in which the probability density (and the norm) remains conserved. ${ }^{29}$ Be this as it may, it has always been recognized that such states contain useful physical information. For example, the temporal decay of the resonant state 
corresponding to a bound state in the zero field limit is related to the life time of that state under the influence of the field.

The mathematical peculiarities were perhaps perceived less as an obstacle in electromagnetics where the counterparts of quantum resonances are the so-called leaky modes. These are configurations of the electromagnetic fields inside unstable resonators and waveguides that can "survive" for long times, but eventually radiate their energy away. They have been studied with keen interest in resonator cavities, ${ }^{30,31}$ optical waveguides, ${ }^{32}$ and photonic ${ }^{33}$ and plasmonic ${ }^{34,35}$ structures and are often utilized for numerical simulations.

However, despite their long history of utility (e.g., Refs. 36-39) in various fields, the unstable states, both quantum and electromagnetic, have not yet been fully understood. On the quantum side, the lack of a general theory for non-self adjoint operators is challenging and is the reason why non-Hermitian systems are mostly investigated on a case-by-case basis (see, e.g., Ref. 25). Ours is precisely such a study of a concrete quantum system.

Our particular motivation for investigating this problem comes from our long time involvement in the problem of high intensity optical pulse propagation. ${ }^{40,41}$ For such high intensity fields, it is very challenging to come up with a material response theory that is reasonably accurate and also reasonably fast to evaluate. This last requirement is of paramount importance for long distance propagation ${ }^{42}$ and rules out any scheme involving a direct integration of the Schrödinger equation ${ }^{43}$ because of the large spectrum of space and time scales involved. There are roughly three orders of magnitude between the oscillation time scale for the electrons in the atom and the optical time scale. This difference, which is a problem for any direct integration scheme, also presents an opportunity for simplification; if one can find a complete set of resonant states for an atom in a constant field, then the solutions for a variable field can be expanded, to a good approximation, in the same set of resonant states by simply making the parameter representing the field strength time dependent. This is an adiabatic assumption that becomes more accurate with the larger the gap between electronic time scale and the optical time scale becomes.

The purpose of this paper is to demonstrate the completeness of the resonant eigenstates for a quantum particle in a square-well potential that is exposed to a homogeneous external electric field and to investigate the convergence properties of the corresponding resonant-state expansion. The external field, even a weak one, has a profound effect on the energetic spectrum of the system; As soon as the field is switched on, all discrete-energy eigenstates dissolve into the energy-continuum which fills up the whole real axis. ${ }^{44}$ At the same time, resonances appear in the complex energy plane, and it is these decaying states our work is concerned with. The results presented in this work generalize and extend the findings in our previous study, ${ }^{45}$ where the convergence of a resonant state expansion was investigated for the case of a zero-range Dirac-delta potential. ${ }^{46-48}$ In particular, the more realistic system investigated here allows us to make conjectures concerning a wide family of one-dimensional quantum systems.

The paper is organized as follows. In Sec. II, we set up the problem by introducing the resonant states for a square-well potential and the locations of their energy eigenvalues in the complex plane; these results are known from the literature. ${ }^{49}$ In an attempt to make the paper more readable, we decided to omit most of the technical but rather standard calculation details in favor of demonstrating the validity of important intermediate results with the help of high-precision numerical tests. In particular, we have made extensive numerical investigations into the convergence of the resonant state expansions and in Sec. III we show some of the results of these investigations. They indicate strongly that the resonant state expansions converge point-wise to the left (i.e., against the pull of the field) of the well and inside the well, but diverge to the right of the well, assuming here that the external field points to the right. This result is consistent with what was obtained for the case of a Dirac delta potential in Ref. 45. In Sec. IV, we prove that what the numerical evidence indicates is indeed true. Resonant state expansions are shown to converge point-wise to the left of the well and inside the well and diverge to the right of the well for all reasonable initial data for the Schrödinger equation. In Sec. V, we investigate the asymptotic form of the terms in the resonant state expansion and make precise statements about the rate of convergence and how this rate relates to the smoothness of the function that is being expanded. We also present high-precision numerical calculations to verify the correctness of our asymptotic formulas and the statements on convergence rates. One surprising 
conclusion that came out of this investigation is that the depth of the potential well does not play much of a role; both the question of convergence of the resonant state expansion and its asymptotic rate of convergence are independent of the depth of the well. We finish the paper with Sec. VI where we briefly discuss on what has been achieved and where we also spell out the aforementioned conjecture in some more detail.

\section{STARK RESONANT STATES FOR A SQUARE WELL}

Let us consider the following Hamiltonian:

$$
H=-\frac{1}{2} \partial_{x x}+V(x)-\varepsilon x,
$$

where $\varepsilon$ is the strength of the external field and where we without loss of generality assume that $\varepsilon>0$, which corresponds to the external electric field pulling the electron to the right. The atomic potential, $V(x)$, is modeled by a square well of width $2 d$ and depth $V_{0}$

$$
V(x)= \begin{cases}-V_{0}, & |x|<d \\ 0, & |x|>d\end{cases}
$$

According to Siegert's characterization, Stark resonant states for the square well are wave functions of the form

$$
\psi(x, t)=\psi_{\omega}(x) e^{-i \omega t}
$$

where $\psi_{\omega}(x)$ are solutions to the equation

$$
H \psi_{\omega}=\omega \psi
$$

which satisfy the boundary conditions

$$
\begin{aligned}
& \psi_{\omega}(x) \rightarrow 0 \text { when } x \rightarrow-\infty, \\
& \psi_{\omega}(x) \text { and } \psi_{\omega}^{\prime}(x) \text { are continuous at } x=-d, d, \\
& \psi_{\omega}(x) \text { is a purely outgoing wave at } x=\infty .
\end{aligned}
$$

The resonant states can be expressed in terms of Airy functions in the form

$$
\psi_{p}(x) \equiv \psi_{\omega_{p}}(x)= \begin{cases}a_{1} \operatorname{Ai}\left(y_{1}\left(x, \omega_{p}\right)\right) & x<-d, \\ a_{2} \operatorname{Ai}\left(y_{2}\left(x, \omega_{p}\right)\right)+a_{3} \operatorname{Bi}\left(y_{2}\left(x, \omega_{p}\right)\right), & -d<x<d, \\ a_{4} \operatorname{Ci}^{+}\left(y_{1}\left(x, \omega_{p}\right)\right) & d<x<\infty,\end{cases}
$$

with the notation $\mathrm{Ci}^{+}=\mathrm{Bi}+i \mathrm{Ai}$ representing an Airy combination that asymptotically behaves as an outgoing wave. The functions $y_{1}(x, \omega)$ and $y_{2}(x, \omega)$ that parametrize the arguments of the Airy functions above are given by

$$
\begin{aligned}
& y_{1}(x, \omega)=-2(2 \varepsilon)^{-\frac{2}{3}}(\varepsilon x+\omega), \\
& y_{2}(x, \omega)=-2(2 \varepsilon)^{-\frac{2}{3}}\left(\varepsilon x+V_{0}+\omega\right) .
\end{aligned}
$$

The resonant eigenvalues $\omega_{p}$ are obtained as solvability conditions for the coefficients $a_{i}$ ensuring that the wave function and its derivative are both continuous at the edges of the potential well, at $x= \pm d$. There is a countable set of such solutions in the complex plane, determined by the equation

$$
\operatorname{det} M\left(\omega_{p}\right)=0 \text {, }
$$

where $M(\omega)$ is a certain $4 \times 4$ matrix. The explicit expression for the determinant of $M(\omega)$ is

$$
\operatorname{det} M(\omega)=\left(A_{0} A_{1}^{\prime}-A_{0}^{\prime} A_{1}\right)\left(B_{2} C_{3}^{\prime}-B_{2}^{\prime} C_{3}\right)-\left(A_{0} B_{1}^{\prime}-A_{0}^{\prime} B_{1}\right)\left(A_{2} C_{3}^{\prime}-A_{2}^{\prime} C_{3}\right),
$$

where we have defined

$$
\begin{aligned}
& A_{0}=\operatorname{Ai}\left(\mu\left(-d+\frac{\omega}{\varepsilon}\right)\right), A_{1}=\operatorname{Ai}\left(\mu\left(-d+\frac{\omega+V_{0}}{\varepsilon}\right)\right), B_{1}=\operatorname{Bi}\left(\mu\left(-d+\frac{\omega+V_{0}}{\varepsilon}\right)\right), \\
& A_{2}=\operatorname{Ai}\left(\mu\left(d+\frac{\omega+V_{0}}{\varepsilon}\right)\right), B_{2}=\operatorname{Bi}\left(\mu\left(d+\frac{\omega+V_{0}}{\varepsilon}\right)\right), C_{3}=\mathrm{Ci}^{+}\left(\mu\left(d+\frac{\omega}{\varepsilon}\right)\right)
\end{aligned}
$$

with $\mu=-(2 \varepsilon)^{\frac{1}{3}}$. 
The resonant eigenvalues lie in the lower complex half plane which means that the corresponding resonant-state wave functions (2) are decaying in time, as they should. Figure 1 shows the zero contours of the real and imaginary parts of the equation. The points where they cross are the zero points of the determinant and thus are the resonant eigenvalues. There exist two infinite families of resonant eigenvalues. The family on the right side of the imaginary axis, called as the A-series in this paper, has zeros located close to the positive real axis and correspond to longer living states, while the family to the left of the imaginary axis has eigenvalues located along the $\operatorname{ray} \arg (z)=-\frac{2 \pi}{3}$ and corresponds to fast decaying states. We call this second family as the $\mathrm{C}$-series. Figure 1 also shows a finite family of resonant states lying close to the negative real axis that corresponds to the bound state eigenvalues for the square well in the limit when the external field approach zero. We call these states as perturbed bound states. This structural division of the resonant states into a finite number of perturbed bound states and the two infinite A-series and C-series is also seen for the short range delta potential in Ref. 45, is probably generic, and should be expected for more general potentials also.

Our focus in this paper is to investigate to what extent the resonant states we have found can be used to expand initial conditions for the Schrödinger equation. If any given initial condition can be expanded in resonant states

$$
f(x)=\sum_{p} \frac{\left(f, \psi_{p}\right)}{\left(\psi_{p}, \psi_{p}\right)} \psi_{p}(x),
$$

the solution to the time dependent Schrödinger equation for the Hamiltonian operator (1), with initial data $\psi(x, 0)=f(x)$, is given by

$$
\psi(x, t)=\sum_{p} \frac{\left(f, \psi_{p}\right)}{\left(\psi_{p}, \psi_{p}\right)} \psi_{p}(x) e^{-i \omega_{p} t} .
$$

This is an exact solution for a static external field corresponding to a fixed value for $\varepsilon$ in (1). For the case of an external field that varies slowly on the atomic time scale, which we take to be atto-seconds, the expansion (8), where now the resonant states and their complex eigenvalues vary in time through their dependence on $\varepsilon=\varepsilon(t)$, is a good approximation to the exact solution.

The eigenvalue problem (3) and (4) is not self-adjoint and as a consequence the eigenvalues displayed in Fig. 1 are complex and the resonant states (5) are not normalizable on the real line. This is, as discussed in the Introduction, to be expected on physical grounds.

In order to achieve the normalization for the resonant states, we rely on the technique used in Ref. 50. In this technique, one introduces a complex contour $\mathcal{L}$ on which the resonant states are

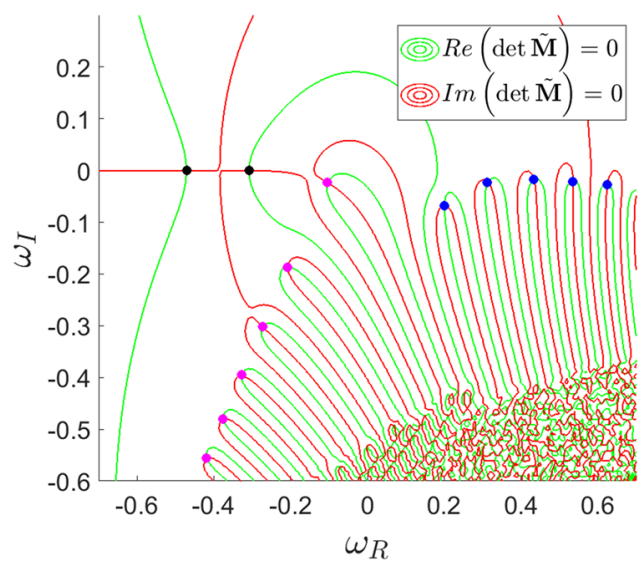

FIG. 1. Contour plot of det $M\left(\omega_{R}+i \omega_{I}\right)$. The green and red lines are the zero contours of the real and imaginary part of det $M\left(\omega_{R}+i \omega_{I}\right)$. The parameters chosen for this illustration were $\varepsilon=0.03, V_{0}=0.5, d=4$. The eigenvalues from the A-series are the blue dots, those from the $\mathrm{C}$-series are purple, and the perturbed bound states are in black. 
evaluated. The contour we will be using has the form

$$
\mathcal{L}=z(x)= \begin{cases}x, & x<x_{c}, \\ x_{c}+e^{i \theta}\left(x-x_{c}\right) & x>x_{c}\end{cases}
$$

which serves its purpose for any chosen parameters $x_{c}>0$ and $0<\theta \leq \frac{\pi}{2}$. In the following, we will consider $\theta=\frac{\pi}{2}$ and $x_{c}>d$ to the right of the well. One can see from the form of the resonant states that they decay on the negative axis, while on the positive part, where the outgoing wave dominates the wave function shape, they show exponential growth. It turns out that for any $0<\theta \leq \frac{\pi}{2}$, the outgoing part of the wave function decays along the contour and integration along the contour can be used to normalize the states. The normalization is achieved by replacing the usual Hermitian inner product for complex valued functions on the real line by another bilinear product for complex valued functions defined on the contour $\mathcal{L}$; this is the product denoted by $(\cdot, \cdot)$ in $(7)$ and (8).

More generally, for any complex contour $C$ and analytic functions $\Phi$ and $\Psi$, we can define a bilinear complex valued product $(\Phi, \Psi)$ by the formula

$$
(\Phi, \Psi)=\int_{C} d z \Phi(z) \overline{\Psi(z)},
$$

where $\bar{\Psi}(z)=\overline{\Psi(\bar{z})}$ is the natural generalization of a complex conjugate preserving the class of analytic functions. For the particular choice of contour (9) with $\theta=\frac{\pi}{2}$, we have the explicit expression

$$
(\varphi, \psi)=\int_{-\infty}^{x_{c}} d x \varphi(x) \psi(x)+i \int_{x_{c}}^{\infty} d x \varphi(x) \psi(x),
$$

where now $\varphi(x)=\Phi(z(x))$ and $\psi(x)=\overline{\Psi(z(x))}$ are smooth functions defined on the real axes that are singular at the point $x=x_{c}$. At the point $x_{c}$, we have

$$
\begin{aligned}
& \left.\partial_{x} \varphi\right|^{a}=\left.i \partial_{x} \varphi\right|_{a}, \\
& \left.\partial_{x} \psi\right|^{a}=\left.i \partial_{x} \psi\right|_{a},
\end{aligned}
$$

where for any function $f(x)$ we have introduced the notation $\left.f\right|^{x_{c}}=\lim _{x \rightarrow x_{c}^{+}}$and $\left.f\right|_{x_{c}}=\lim _{x \rightarrow x_{c}^{-}} f(x)$. This set of functions clearly forms a vector space over the complex numbers, but it is an unusual complex vector space in several ways. Taking the complex conjugate of vectors in $V$ brings us out of the space, the product, $(\cdot, \cdot)$, is not positive, $(\varphi, \varphi)$ is in general a complex number, and this complex valued product is symmetric $(\varphi, \psi)=(\psi, \varphi)$. However, the space $V$ equipped with the product $(10)$ is the natural setting for working with resonant states; not only does it contain the resonant states as vectors but also the Hamiltonian (1) is self-adjoint on this space

$$
(H \varphi, \psi)=(\varphi, H \psi) .
$$

This means that resonant states corresponding to different eigenvalues are orthogonal and this fact leads to the expansion (1). For self-adjoint operators in Hilbert space, one has a completeness theorem. We are not aware of the existence of such a result for the space $V$. In order to prove such a result for the space $V$, one has first to construct an appropriate topology and then use this topology as a starting point for a completeness theorem. This kind of investigation, which certainly is worthwhile to pursue mathematically, will however at best to lead to some kind of convergence that is weaker than the pointwise convergence which is our goal to investigate in the current paper. We will therefore not pursue these mathematical issues here.

Note that the relevance of this kind of mathematical structure for describing expansion into decaying states was noted already in 1938 by Kapur and Peierls while studying the dispersion formulas for nuclear reactions. ${ }^{51}$

\section{NUMERICAL RESULTS}

We have done extensive numerical tests of the resonant state expansions (7). For particular choices of functions, $f(x)$, and particular square wells, the expansion coefficients were calculated 


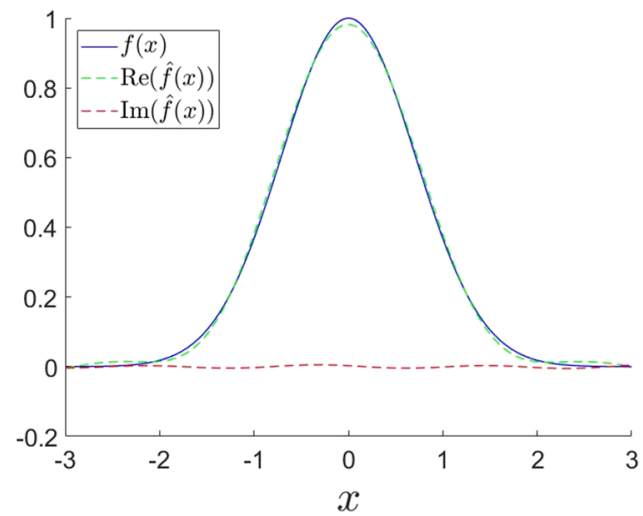

(a)

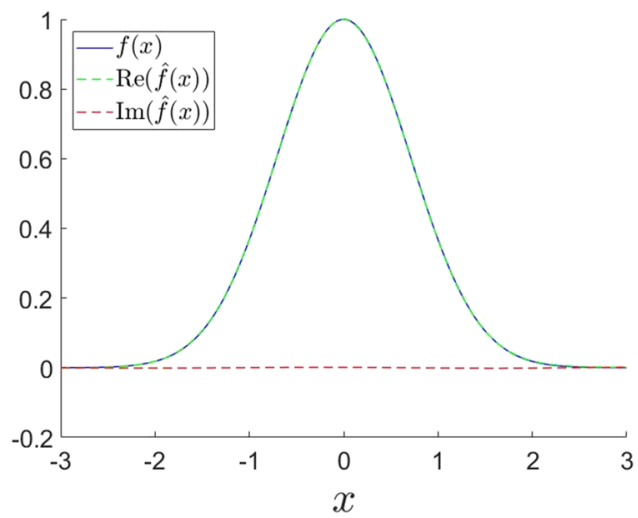

(b)

FIG. 2. The test function $f(x)=e^{-x^{2}}$ and the corresponding expansion $\hat{f}(x)$ using resonant states from A-series. The parameters are $V_{0}=2, d=14, \varepsilon=0.03$. Note that the function to be expanded in this and the following figure is well localized inside the potential well. (a) 100 terms used in the expansion. (b) 1000 terms used in the expansion.

using resonant states from the set of perturbed bound states and the A-series. The sample results displayed in Figs. 2 and 3 show what we find is generic behaviour. For this illustration, we used a Gaussian function and a Gaussian wave packet, and computed from 20 to 1000 terms taken from the set of perturbed bound states and from the A-series in the resonant state expansion thereby defining a function $\hat{f}(x)=\sum_{n} c_{n} \psi_{\omega_{n}}(x)$, which could possibly be different from our original $f$. We calculate numerically, the complex energy eigenvalues, $\omega_{p}$, which are solutions of (6) and the coefficients $a_{1} \cdots a_{4}$, ensuring the continuity of the resonant states at the points $x=-d, d$. From these pictures and many like them, our conjecture is that resonant state expansions based on the set of perturbed bound states and the A-series will converge point-wise for all reasonable initial data for the Schrödinger equation. The convergence appears to be fairly slow compared to the convergence of a regular Fourier series for the same functions. The slow convergence is, in particular, well illustrated by Fig. 3. The correspondence between the convergence and the pictures in Fig. 3 lies in the fact that if we increase the number of terms in the expansion, the difference between the two functions decreases.

In the remainder of this paper, we will prove that our conjecture is true and also investigate in detail how the rate of convergence depends on all parameters in the problem.

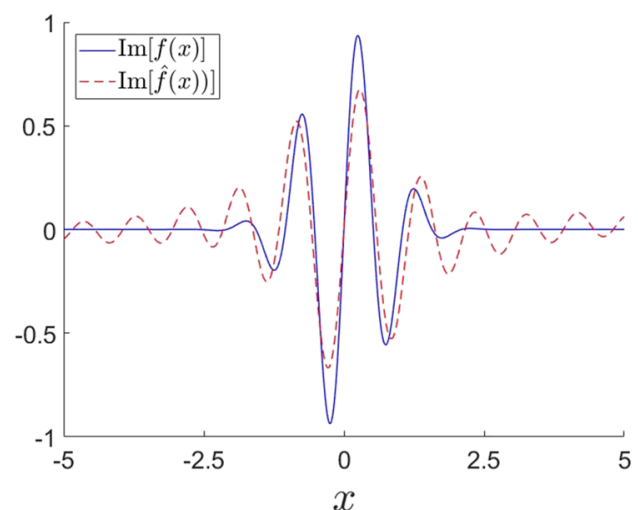

(a)

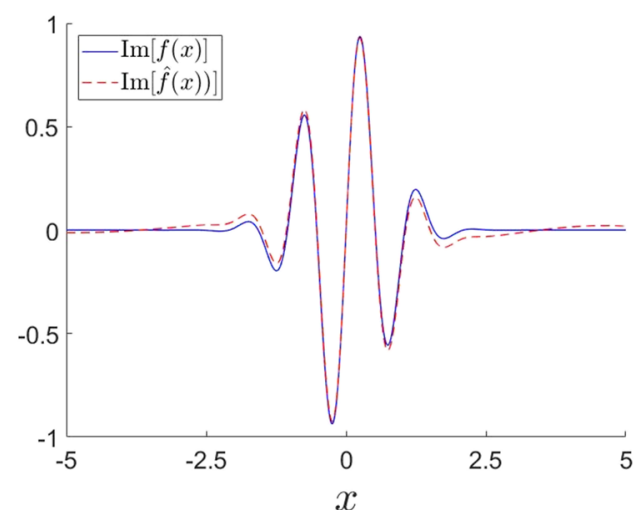

(b)

FIG. 3. The test function $f(x)=e^{-x^{2}} e^{i 6 x}$ and the corresponding expansion $\hat{f}(x)$ using resonant states from A-series. The parameters are $V_{0}=2, d=14, \varepsilon=0.03$. (a) 1000 terms used in the expansion. (b) 10086 terms used in the expansion. 


\section{RESONANT STATES EXPANSION}

The numerical tests in Sec. III suggest that the resonant state expansion (7) converges inside the well, although the rate of convergence is rather slow. In this chapter, we present a proof that confirms the pointwise convergence. Let us begin by noting that the Stark Hamiltonian (1) has no bound states. The continuous spectrum is found by imposing scattering boundary conditions on the Stark Hamiltonian where we have both incoming and outgoing waves at positive infinity and decaying outgoing waves at negative infinity.

The Stark Hamiltonian is an unbounded self-adjoint operator and as such it has an associated resolution of the identity. ${ }^{52}$ In general, the spectral resolution contains an integration over point spectra and also absolute continuous and singularly continuous spectral components. For the Stark Hamiltonian in this paper, it has been proven ${ }^{53-55}$ that both the point spectrum and the singularly continuous spectrum are empty and that the absolutely continuous spectrum is equal to the whole real line. Thus we have

$$
\int_{-\infty}^{\infty} \psi_{\omega}(x) \psi_{\omega}\left(x^{\prime}\right) \mathrm{d} \omega=\delta\left(x-x^{\prime}\right)
$$

where the complex conjugate in (11) is missing because in our case the scattering states are real. For our particular Stark Hamiltonian (1), we find that the scattering states are given by

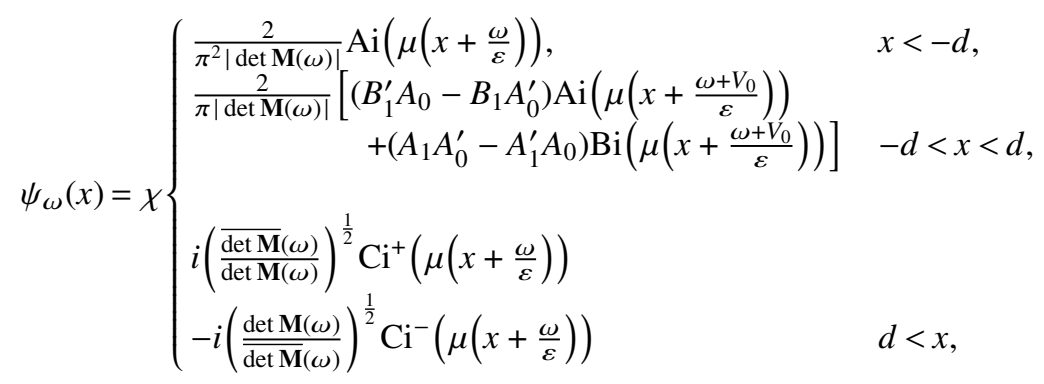

where $\mu=-(2 \varepsilon)^{\frac{1}{3}}, \mathrm{Ci}^{ \pm}=\operatorname{Bi}(x) \pm i \operatorname{Ai}(x)$ and where $\overline{\operatorname{det} M}(\omega)=\overline{\operatorname{det} M(\bar{\omega})}$. The normalization constant $\chi=2^{-\frac{2}{3}} \varepsilon^{-\frac{1}{6}}$ ensures that the multiplier of the delta functions on the right-hand side of (11) is one.

Since $\mathrm{Ci}^{+}$and $\mathrm{Ci}^{-}$represent outgoing and incoming waves at positive infinity, there is a natural split of the scattering states in outgoing, $\psi_{\omega}^{+}(x)$, and incoming, $\psi_{\omega}^{-}(x)$, parts. These are

$$
\begin{aligned}
& \psi_{\omega}^{+}(x)=\chi \begin{cases}i\left(\frac{\operatorname{det} \mathbf{M}(\omega)}{\operatorname{det} \mathbf{M}(\omega)}\right)^{\frac{1}{2}} \mathrm{Ci}^{+}\left(\mu\left(d+\frac{\omega}{\varepsilon}\right)\right) \frac{\operatorname{Ai}\left(\mu\left(x+\frac{\omega}{\varepsilon}\right)\right)}{\pi p(\omega)} & x<-d, \\
i\left(\frac{\operatorname{det} \mathbf{M}(\omega)}{\operatorname{det} \mathbf{M}(\omega)}\right)^{\frac{1}{2}} \mathrm{Ci}^{+}\left(\mu\left(d+\frac{\omega}{\varepsilon}\right)\right) \frac{1}{p(\omega)} & \\
{\left[\left(B_{1}^{\prime} A_{0}-B_{1} A_{0}^{\prime}\right) \operatorname{Ai}\left(\mu\left(x+\frac{\omega+V_{0}}{\varepsilon}\right)\right)\right.} & \\
\left.+\left(A_{1} A_{0}^{\prime}-A_{1}^{\prime} A_{0}\right) \operatorname{Bi}\left(\mu\left(x+\frac{\omega+V_{0}}{\varepsilon}\right)\right)\right], & -d<x<d, \\
i\left(\frac{\operatorname{det} \mathbf{M}(\omega)}{\operatorname{det} \mathbf{M}(\omega)}\right)^{\frac{1}{2}} \mathrm{Ci}^{+}\left(\mu\left(x+\frac{\omega}{\varepsilon}\right)\right) & d<x,\end{cases} \\
& \psi_{\omega}^{-}(x)=\chi \begin{cases}-i\left(\frac{\operatorname{det} \mathbf{M}(\omega)}{\operatorname{det} \mathbf{M}(\omega)}\right)^{\frac{1}{2}} \mathrm{Ci}^{-}\left(\mu\left(d+\frac{\omega}{\varepsilon}\right)\right) \frac{\operatorname{Ai}\left(\mu\left(x+\frac{\omega}{\varepsilon}\right)\right)}{\pi p(\omega)} & x<-d, \\
-i\left(\frac{\operatorname{det} \mathbf{M}(\omega)}{\operatorname{det} \mathbf{M}(\omega)}\right)^{\frac{1}{2}} \mathrm{Ci}^{-}\left(\mu\left(d+\frac{\omega}{\varepsilon}\right)\right) \frac{1}{p(\omega)} & \\
{\left[\left(B_{1}^{\prime} A_{0}-B_{1} A_{0}^{\prime}\right) \operatorname{Ai}\left(\mu\left(x+\frac{\omega+V_{0}}{\varepsilon}\right)\right)\right.} & \\
\left.+\left(A_{1} A_{0}^{\prime}-A_{1}^{\prime} A_{0}\right) \operatorname{Bi}\left(\mu\left(x+\frac{\omega+V_{0}}{\varepsilon}\right)\right)\right] & -d<x<d, \\
-i\left(\frac{\operatorname{det} \mathbf{M}(\omega)}{\operatorname{det} \mathbf{M}(\omega)}\right)^{\frac{1}{2}} \operatorname{Ci}^{-}\left(\mu\left(x+\frac{\omega}{\varepsilon}\right)\right) & d<x,\end{cases}
\end{aligned}
$$


where we have defined $p(\omega)=\left(B_{1}^{\prime} A_{0}-B_{1} A_{0}^{\prime}\right) A_{2}+\left(A_{1} A_{0}^{\prime}-A_{1}^{\prime} A_{0}\right) B_{2}$. Observe that by construction, we have

$$
\psi_{\omega}(x)=\psi_{\omega}^{+}(x)+\psi_{\omega}^{-}(x)
$$

We can use the completeness to split any functions in the span of $\left\{\psi_{\omega}\right\}$ into outgoing and incoming parts

$$
\begin{aligned}
f(x) & =\int_{-\infty}^{\infty} \mathrm{d} s \delta(x-s) f(s) \\
& =\int_{-\infty}^{\infty} \mathrm{d} s \int_{-\infty}^{\infty} \mathrm{d} \omega \psi_{\omega}(x) \psi_{\omega}(s) \int_{-\infty}^{\infty} \mathrm{d} \omega^{\prime} a\left(\omega^{\prime}\right) \psi_{\omega^{\prime}}(s) \\
& =\int_{-\infty}^{\infty} \mathrm{d} \omega^{\prime} a\left(\omega^{\prime}\right) \int_{-\infty}^{\infty} \mathrm{d} \omega \psi_{\omega}(x) \int_{-\infty}^{\infty} \mathrm{d} s \psi_{\omega}(s) \psi_{\omega^{\prime}}(s),
\end{aligned}
$$

where $a\left(\omega^{\prime}\right)=\int_{-\infty}^{\infty} \mathrm{d} x \psi_{\omega^{\prime}}(x) f(x)$ is the energy representation of $f(x)$. We now split the scattering state $\psi_{\omega^{\prime}}(s)$ into outgoing and incoming parts using (12) and (13) and find that $f(x)=f^{+}(x)+f^{-}(x)$, where

$$
f^{ \pm}(x)=\int_{-\infty}^{\infty} \mathrm{d} \omega^{\prime} a\left(\omega^{\prime}\right) \int_{-\infty}^{\infty} \mathrm{d} \omega \psi_{\omega}(x) \int_{-\infty}^{\infty} \mathrm{d} s \psi_{\omega}(s) \psi_{\omega^{\prime}}^{ \pm}(s)
$$

Using special rules for anti-derivatives of Airy functions ${ }^{56}$ and generalizing the approach used in Ref. 45, we get the following expressions for the outgoing and incoming parts of $f(x)$ :

$$
f^{ \pm}(x)=\frac{1}{2} \int_{-\infty}^{\infty} \mathrm{d} \omega^{\prime} a\left(\omega^{\prime}\right)\left[\mp i \frac{\left|\operatorname{det} \mathbf{M}\left(\omega^{\prime}\right)\right|}{p\left(\omega^{\prime}\right)}\right] \int_{-\infty}^{\infty} \mathrm{d} \omega \psi_{\omega}(x) \frac{\psi_{\omega}(d)}{\omega-\omega^{\prime} \mp i \xi} .
$$

Note that the inner integral in (14) has been regularized using a parameter $\xi$ that will be removed at the end of our calculations by letting it approach zero from above.

We will now focus on the outgoing part of $f(x)$; the incoming part of $f(x)$ is treated in an entirely similar manner. For the outgoing part of $f(x)$, we observe that the integrand in the inner integral in the expression (14) defining $f^{+}(x)$

$$
P_{\xi}\left(\omega^{\prime}\right)=\int_{-\infty}^{\infty} \mathrm{d} \omega \psi_{\omega}(x) \frac{\psi_{\omega}(d)}{\omega-\omega^{\prime}-i \xi}
$$

has poles at the point $\omega=\omega^{\prime}+i \xi$ in the upper half plane and at the zeroes for the functions det $M(\omega)$ and $\overline{\operatorname{det} M}(\omega)$ in the lower and upper half plane.

We now rewrite the quantity $P_{\xi}\left(\omega^{\prime}\right)$ using the residue theorem on the contour $\Gamma_{R}$ which is closed in the lower half plane. This contour is depicted in Fig. 4. The only poles of the integrand inside this contour are the zeroes of det $M(\omega)$ and we thus have

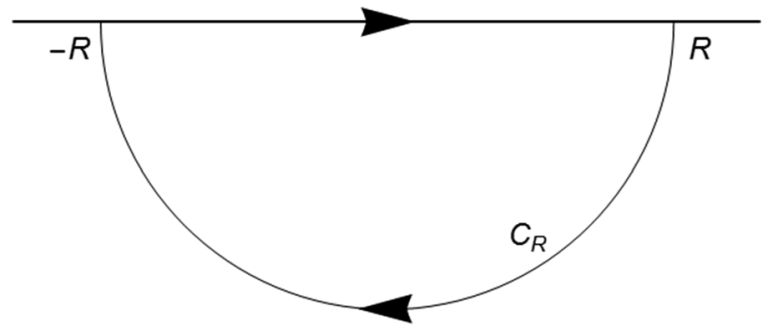

FIG. 4. Closed contour $\Gamma_{R}$ in the lower complex frequency half plane, $\omega$. 


$$
\begin{aligned}
P_{\xi}^{R}\left(\omega^{\prime}\right) & \equiv \int_{-R}^{R} \mathrm{~d} \omega \psi_{\omega}(x) \frac{\psi_{\omega}(d)}{\omega-\omega^{\prime}-i \xi} \\
& =\frac{2 \pi}{i} \sum_{j} \operatorname{Res}\left(\psi_{\omega}(x) \frac{\psi_{\omega}(d)}{\omega-\omega^{\prime}-i \xi}, \omega_{j}\right)-\int_{C_{R}} \mathrm{~d} \omega \psi_{\omega}(x) \frac{\psi_{\omega}(d)}{\omega-\omega^{\prime}-i \xi}
\end{aligned}
$$

Observe that $P_{\xi}^{R}$ converges to $P_{\xi}$ in (15) as $R \rightarrow \infty$. From (16), it is evident that if the integral over the contour $C_{R}$ vanishes in the limit when $R$ approaches infinity

$$
\lim _{R \rightarrow \infty} \int_{C_{R}} \mathrm{~d} \omega \psi_{\omega}(x) \frac{\psi_{\omega}(d)}{\omega-\omega^{\prime}-i \xi}=0
$$

then the $P_{\xi}\left(\omega^{\prime}\right)$ will be a sum of residues

$$
P_{\xi}\left(\omega^{\prime}\right)=\frac{2 \pi}{i} \sum_{j} \operatorname{Res}\left(\psi_{\omega}(x) \frac{\psi_{\omega}(d)}{\omega-\omega^{\prime}-i \xi}, \omega_{j}\right),
$$

and from this it is then a simple matter to show that the regulator $\xi$ can be safely removed and that formula (14) reduces to a resonant state expansion for the outgoing part of $f$

$$
f^{+}(x)=\sum_{j} c_{j} \psi_{j}(x)
$$

which is what we wanted to prove.

Thus the proof of convergence for the resonant state expansion for any given $x$ is reduced to showing that the limit (17) holds for that $x$. In order to investigate this limit, we parameterize the circular $\operatorname{arc} C_{R}$ in the lower half plane using $\omega=R e^{i \theta}$ and use standard formulas for the Airy functions ${ }^{57}$ in the large argument limit to derive asymptotic formulas for the scattering states $\psi_{\omega}$ in the limit of large $R$. Because of the well known Stokes phenomenon, the resulting asymptotic expressions for the scattering states are different for the two angular sectors $-\frac{2 \pi}{3}<\theta<0$ and $-\pi<\theta<-\frac{2 \pi}{3}$. We find that in the second sector, the scattering state $\psi_{\omega}(x)$ decays exponentially for all $x$ and thus this angular sector gives no contribution to the limit (17). In the first angular sector, we have the following asymptotic expression for the scattering state:

$$
\psi_{\omega}(x)=\chi \begin{cases}\frac{2(\kappa R)^{\frac{1}{4}}}{i \pi^{\frac{1}{2}} \sigma^{\frac{1}{2}}} e^{-i \beta \varrho R^{\frac{1}{2}}} e^{\varpi x R^{\frac{1}{2}}} & x<-d, \\ \frac{2(\kappa R)^{\frac{1}{4}}}{i \pi^{\frac{1}{2}} \sigma^{\frac{1}{2}}} e^{-i(\beta+\sigma) \varrho R^{\frac{1}{2}}} e^{\varpi R^{\frac{1}{2}}\left(x+\frac{V_{0}}{\varepsilon}\right)} & -d<x<d, \\ -\frac{\sigma^{\frac{1}{2}}}{2(\kappa R)^{\frac{3}{4}} \pi^{\frac{1}{2}}} e^{i \varrho R^{\frac{1}{2}}(\sigma-3 \beta)} e^{-\varpi R^{\frac{1}{2}}\left(x+\frac{V_{0}}{\varepsilon}\right)} & \\ i \frac{2(\kappa R) \frac{1}{4}}{\pi^{\frac{1}{2}} \sigma^{\frac{1}{2}}} e^{-i \beta \varrho R^{\frac{1}{2}}} e^{\varpi x R^{\frac{1}{2}}}-i \frac{\sigma^{\frac{1}{2}}}{2 \pi^{\frac{1}{2}}(\kappa R)^{\frac{3}{4}}} e^{i \beta \varrho R^{\frac{1}{2}}} e^{-\varpi x R^{\frac{1}{2}}} & d<x,\end{cases}
$$

where $\varpi, \varrho$ are complex numbers depending on $\theta$ and $\mu=(2 \varepsilon)^{-\frac{2}{3}}, \beta=2 \mu \varepsilon d, \sigma=2 \mu V_{0}$, and $\kappa=2 \mu e^{i \theta}$. Let us start analyzing the various regions in $x$. For $x<-d$, we find that the limit is zero and that for $x>d$ it does not exist. Thus the resonant state expansion converges for $x<-d$, but not for $x>d$. This is consistent with what was found for the Dirac delta potential in Ref. 45 if we imagine approaching the Dirac delta function using a sequence of square wells where $V_{0}$ approach minus infinity, while $d$ approach zero. 
For $x$ inside the square well, where $-d<x<d$, the asymptotic expression for the integrand in (17) is found to be

$$
\begin{aligned}
& \psi_{\omega}(x) \frac{\psi_{\omega}\left(d^{+}\right)}{\omega-\omega^{\prime} \mp i \xi} \approx-\frac{\chi^{2}}{\omega-\omega^{\prime} \mp i \xi}\left(\frac{1}{\pi(\kappa R)^{\frac{1}{2}}}\right. \\
& e^{-i \sigma \varrho_{r} R^{\frac{1}{2}}} e^{(2 \mu)^{\frac{1}{2}}\left|\sin \left(\frac{1}{2} \theta\right)\right| R^{\frac{1}{2}}\left[\varepsilon 2 \mu\left(x+\frac{V_{0}}{\varepsilon}-d\right)-\sigma\right]} e^{i \varpi_{i} R^{\frac{1}{2}}\left(x+\frac{V_{0}}{\varepsilon}-d\right)} \\
& -\frac{\sigma}{8 \pi(\kappa R)^{\frac{3}{2}}} \\
& e^{i \varrho_{r} R^{\frac{1}{2}}(\sigma-2 \beta)} e^{(2 \mu)^{\frac{1}{2}}\left|\sin \left(\frac{1}{2} \theta\right)\right| R^{\frac{1}{2}}\left[-\varepsilon 2 \mu\left(x+\frac{V_{0}}{\varepsilon}+d\right)+(\sigma-2 \beta)\right]} e^{-i \varpi_{i} R^{\frac{1}{2}}\left(x+\frac{V_{0}}{\varepsilon}+d\right)} \\
& -\frac{4(\kappa R)^{\frac{1}{2}}}{\pi \sigma} \\
& e^{-i(2 \beta+\sigma) \varrho_{r} R^{\frac{1}{2}}} e^{(2 \mu)^{\frac{1}{2}}\left|\sin \left(\frac{1}{2} \theta\right)\right| R^{\frac{1}{2}}\left[\varepsilon 2 \mu\left(x+\frac{V_{0}}{\varepsilon}+d\right)-(2 \beta+\sigma)\right]} e^{i \varpi_{i} R^{\frac{1}{2}}\left(x+\frac{V_{0}}{\varepsilon}+d\right)} \\
& +\frac{1}{\pi(\kappa R)^{\frac{1}{2}}} \\
& \left.e^{i \varrho_{r} R^{\frac{1}{2}}(\sigma-4 \beta)} e^{(2 \mu)^{\frac{1}{2}}\left|\sin \left(\frac{1}{2} \theta\right)\right| R^{\frac{1}{2}}\left[-\varepsilon 2 \mu\left(x+\frac{V_{0}}{\varepsilon}-d\right)+(\sigma-4 \beta)\right]} e^{-i \varpi_{i} R^{\frac{1}{2}}\left(x+\frac{V_{0}}{\varepsilon}-d\right)}\right) .
\end{aligned}
$$

If this expression decays exponentially in the limit $R \rightarrow \infty$, the resonant state expansion will converge inside the square well. In (19), we have four different terms that need to be checked separately. The first two exponentials in (19) decay if

$$
\begin{array}{rrr}
\varepsilon 2 \mu\left(x+\frac{V_{0}}{\varepsilon}-d\right)-\sigma & <0, & -\varepsilon 2 \mu\left(x+\frac{V_{0}}{\varepsilon}+d\right)+(\sigma-2 \beta)<0, \\
\Downarrow & \Downarrow \\
d>x, & -3 d<x .
\end{array}
$$

These conditions are satisfied inside the well, $-d<x<d$.

The second two parts of (19) converge to zero if

$$
\begin{array}{rrr}
\varepsilon 2 \mu\left(x+\frac{V_{0}}{\varepsilon}+d\right)-(2 \beta+\sigma) & <0, & -\varepsilon 2 \mu\left(x+\frac{V_{0}}{\varepsilon}-d\right)+(\sigma-4 \beta)<0, \\
\Downarrow & \Downarrow \\
d>x, & -3 d<x,
\end{array}
$$

which are also satisfied inside the well. Note that the depth of the well $V_{0}$ disappears from the inequalities (20) and (21). Thus we reach the surprising conclusion that the resonant state expansion converges for all $x$ inside the square well independently of the depth of the well.

\section{RATE OF CONVERGENCE}

In this section, we directly investigate the rate of convergence of the resonant state expansion (18) found to converge for $-\infty<x<d$ in Sec. IV. As we have discussed in Sec. II of this paper, the energy eigenvalues can be categorized into three groups. The first is the finite set of perturbed bound states, the second set is the A-series, which are located in the fourth quadrant in the complex plane, and the third is the C-series located in the third quadrant. The perturbed set of bound states is finite and thus does not contribute to the rate of convergence, but the A-series and the $\mathrm{C}$-series are both infinite and they do contribute to the rate of convergence. First, we look at the part of the sum corresponding to the eigenvalues from the A-series and after that briefly sum up the results for the $\mathrm{C}$-series. In order to do this, we need an asymptotic expression for resonant state eigenvalues from the A-series. In principle, we find this expression by first finding the leading order contribution of the determinant function, here denoted by det $M^{\infty}(\omega)$, for $|\omega| \rightarrow \infty$ and then finding the leading order 
expression for the roots of the equation det $M^{\infty}(\omega)=0$ that define the A-series. The final formula for the resonant eigenvalues $\omega_{p}$ is simple and is reproduced below in (22), but even though nothing beyond the standard asymptotic formulas for Airy functions are required for this derivation, the details are technical and will not be presented (this remark applies to most of the explicit formulas derived in this section). The formula for $\omega_{p}$ is

$$
\omega_{p} \approx \gamma^{-1}\left(\frac{3 \pi}{2} \xi_{p}\right)^{\frac{2}{3}},
$$

where

$$
\xi_{p}=p-\frac{i}{3 \pi} \ln (p)+i \rho+\frac{i}{2 \pi} \ln \left(\left|\sin \left(c_{2} p^{\frac{1}{3}}\right)\right|\right)-\frac{1}{2 \pi} h(p),
$$

where $p \gg 1$ is the index of the eigenvalues. The parameters appearing in (22) and (23) are given by

$$
\begin{aligned}
\gamma & =2(2 \varepsilon)^{-\frac{2}{3}}, \\
c_{1} & =\frac{V_{0} \gamma}{2^{\frac{1}{3}} 3^{\frac{2}{3}} \pi^{\frac{8}{3}}}, \\
c_{2} & =2 d(3 \pi \varepsilon)^{\frac{1}{3}}, \\
\rho & =\frac{\ln \left(\pi^{2} c_{1}\right)}{2 \pi}, \\
h(p) & =\arg \left(\sin \left(c_{2} p^{\frac{1}{3}}\right)\right) .
\end{aligned}
$$

In Fig. 5, we compare the asymptotic formula for the resonant eigenvalues with an arbitrary precision numerical calculation of the eigenvalues. As we can see, our asymptotic formula is highly accurate.

We will now use the asymptotic formula for the location of the resonant eigenvalues of the A-series to get an estimate of the rate of convergence of the resonant state expansion for functions $f$ whose support are inside the square well. The rate of expansion in general depends on $x$, but here we will focus on the point $x=0$. The resonant state expansion for $f$ then becomes the following numerical series:

$$
\sum_{p} \frac{b_{p}}{N_{p}}
$$

where

$$
\begin{aligned}
& b_{p}=\psi_{\omega_{p}}(0) \int_{-d}^{d} f(x) \psi_{\omega_{p}}(x) \mathrm{d} x, \\
& N_{p}=\int_{-d}^{d} \psi_{\omega_{p}}(x)^{2} \mathrm{~d} x .
\end{aligned}
$$

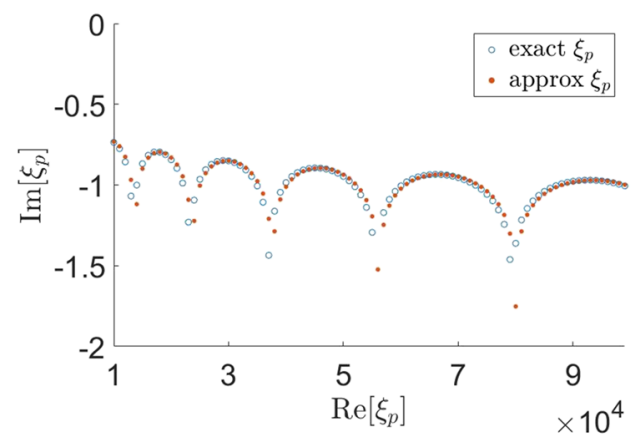

(a)

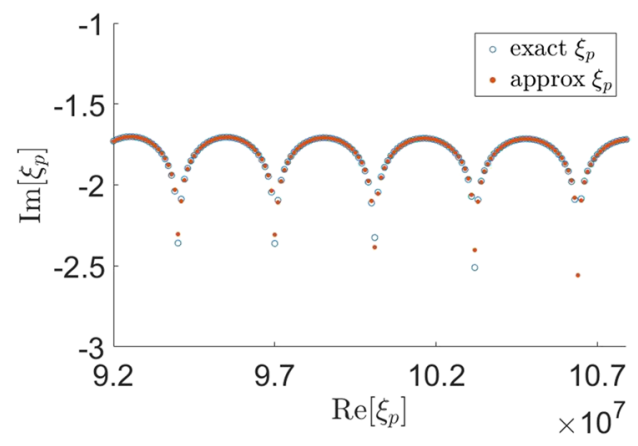

(b)

FIG. 5. Comparison between a high precision numerical calculation of the pole positions and the asymptotic formula (22) for two different ranges of the pole index. 
Using the asymptotic formula for the resonant eigenvalues from the A-series (22), we get the following asymptotic expressions for $b_{p}$ and $N_{p}$ :

$$
\begin{aligned}
& b_{p} \approx \pi^{-1}\left(\frac{3 \pi}{2}\right)^{-\frac{1}{3}} p^{-\frac{1}{3}}\left\{a_{3} \cos \left(g_{1}(p)+\frac{\pi}{4}\right)+a_{2} \sin \left(g_{1}(p)+\frac{\pi}{4}\right)\right\} \\
& \int_{-d}^{d} d x f(x)\left\{a_{3} \cos \left(g_{1}(p)+x g_{2}(p)+\frac{\pi}{4}\right)+a_{2} \sin \left(g_{1}(p)+x g_{2}(p)+\frac{\pi}{4}\right)\right\}, \\
& N_{p} \approx \frac{a_{1}^{2}}{\gamma \varepsilon} \pi^{-1}\left(\frac{3 \pi p}{2}\right)^{\frac{1}{3}}
\end{aligned}
$$

where

$$
\begin{aligned}
& g_{1}(p)=\frac{2}{3} \mu^{\frac{3}{2}} \xi_{p}+\mu^{\frac{1}{2}} p^{\frac{1}{3}} \gamma V_{0}, \\
& g_{2}(p)=\mu^{\frac{1}{2}} \gamma \varepsilon p^{\frac{1}{3}} .
\end{aligned}
$$

Recall that $\left(a_{1}, \ldots, a_{4}\right)$ is a vector that spans the null space of the matrix $M_{p}=M\left(\omega_{p}\right)$. In general, this vector also depends on $p$. However, in the asymptotic range, when $p \gg 1$ the matrix $M_{p}$ simplifies in such a way that the null-space vector can be chosen to be independent of $p$.

In Fig. 6, where we have chosen $f(x)$ to be a Gaussian function, we see how accurate the asymptotic expressions are. They are showing two sets of points, the absolute value of the numerically calculated value of the term, and the value we get from the asymptotic formulas. For smaller values of $p$, the approximation seems to fluctuate around the exact values and does not seem to catch up on the most extreme swings and breaks. However, as we move forward to higher values, the asymptotic formula eventually catches all the wild swings of the terms in the series.

We will now derive some analytic estimates for the rate of convergence using the asymptotic expressions (24) for $b_{p}$ and $N_{p}$. First we note that for the special case of a function that is constant equal to one inside the well and zero outside, we can find an analytic expression for $b_{p}$. Let us call this

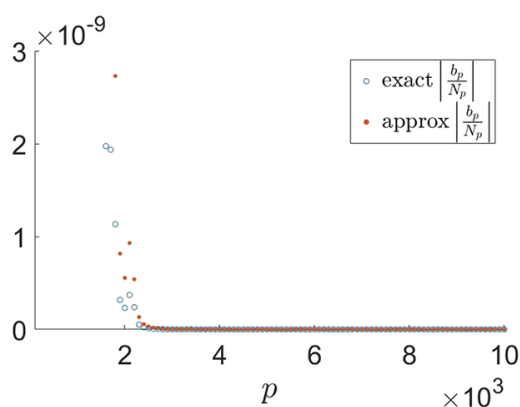

(a)

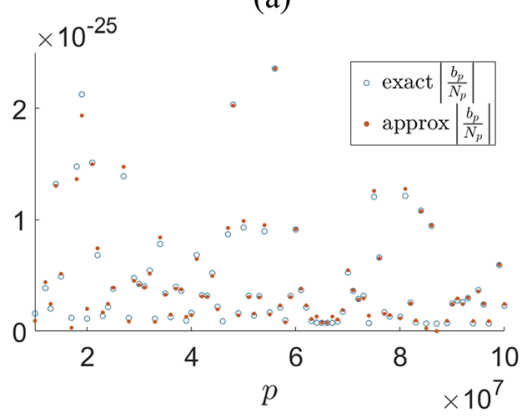

(c)

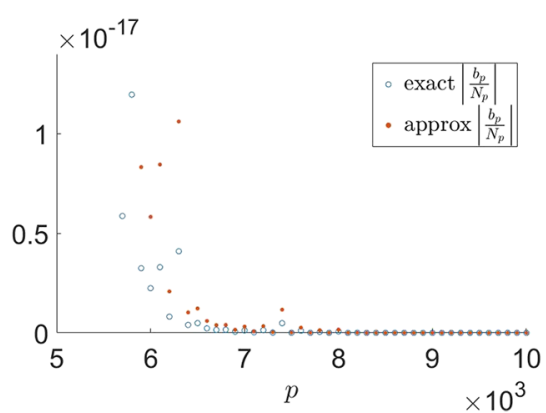

(b)

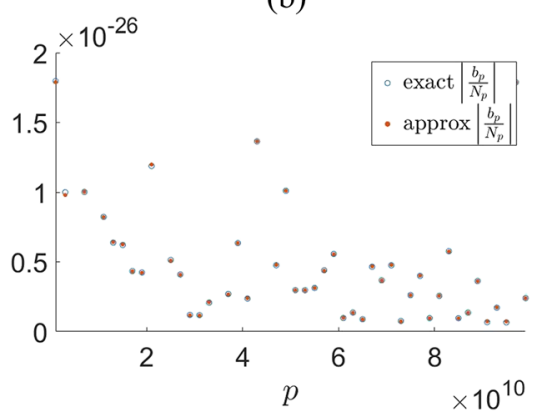

(d)

FIG. 6. The absolute value of exact and asymptotic ratios $\left|\frac{b_{p}}{N_{p}}\right|$ for different ranges of the eigenvalue index $p$. In these pictures, we used $V_{0}=2, d=14, \varepsilon=0.03, x_{c}=20$, and $\theta=\frac{\pi}{2}$. 
quantity as $b_{p}^{0}$. The details of the calculation are relegated to the Appendix. The resulting expression is

$$
\left|b_{p}^{0}\right| \approx \frac{\alpha}{V_{0}\left|\cos \left(d(3 \pi \varepsilon p)^{\frac{1}{3}}\right)\right|},
$$

where $\alpha$ is a numerical factor that does not depend on $p$ or $V_{0}$. Now let $f(x)$ be a function that is zero at $x= \pm d$ and is $n$ times continuously differentiable. From formula (24), it is clear that each time the trigonometric sum under the integral sign is integrated, a factor of $g_{2}(p)$ appears in the denominator. It is also clear that any number of integrations will preserve the form of the trigonometric term in the integrand up to sign after an even number of integrations. Thus, using integration by parts, $n=2 m$ times will give us

$$
\left|\frac{b_{p}}{N_{p}}\right| \lesssim p^{-\frac{n+1}{3}} \frac{\beta M_{n}}{V_{0}\left|\cos \left(d(3 \pi \varepsilon p)^{\frac{1}{3}}\right)\right|},
$$

where $\beta$ is another numerical factor not depending on $p$ or $V_{0}$ and $\left|f^{(n)}(x)\right| \leq M_{n}$ for $x$ inside the well. This formula shows explicitly how the size of the terms in the resonant state series for a function depends on the smoothness of that function and the depth of the well. Note that the depth of the well cancels if we divide two consecutive terms to get the rate of convergence, and we can conclude that the absolute size of the terms depends on the depth of the well as $V_{0}^{-1}$, but that the rate of convergence is independent of $V_{0}$.

So far we have only considered convergence of the part of the resonant state expansion that comes from the A-series. However, detailed investigations of the terms in the series coming from the $\mathrm{C}$-series have shown that they are decaying exponentially in the resonant state index, $p$, and thus have no influence on the question of convergence for the series as a whole. These investigations, which follow the same approach as for the A-series, will not be presented here.

\section{CONCLUSION}

We have investigated the completeness of the Stark resonant states in a system with a square-well potential and a homogeneous external field. Our conclusion is that when the field pulls the particle to the right, the resonant state expansions converge pointwise to the left of the well as well as inside the well. Interestingly, we have found that the existence of convergence is independent of the depth of the potential well, $V_{0}$. In other words, no matter how shallow the well might be, there is a convergent resonant-state expansion.

We have also derived formulas that show how the rate of convergence depends on the smoothness of the function being expanded. These formulas indicate that the rate of convergence is also independent of the depth of the well, but the absolute size of the terms grows like $V_{0}^{-1}$. Thus, for any given target accuracy, a smaller potential depth means that more terms have to be included in the series.

Taking into account the similar nature of the convergence results for the Dirac delta poten$\mathrm{tial}^{45}$ and for the square potential treated in this paper, we conjecture a similar result for a general potential of compact support. Such a potential can be approximated by a finite set of conjoined square wells, as illustrated in Fig. 7, and we expect that our approach can be generalized to this setting using a transfer-matrix technique. In the limit when the set of conjoined square wells approach the smooth potential, there will inevitably be square wells that are arbitrarily shallow but we expect, based on the result derived in this paper, that this will not destroy the convergence. In fact, this same result leads us to conjecture pointwise convergence even for non-compact potentials.

Finally let us note that there are issues that invite further investigations. Our resonant-state expansion is constructed separately for the incoming and outgoing portions of the given wave function, and there is certain similarity here with the Green's function approach in which the imposed boundary conditions can select the outgoing waves. Naturally, what constitutes the outgoing and incoming parts of the given function in the region far from the origin (in the direction of the field) is given and unique, and corresponds to the decomposition into $\mathrm{Ci}^{+}$and $\mathrm{Ci}^{-}$. However, the in- and out-split is not 


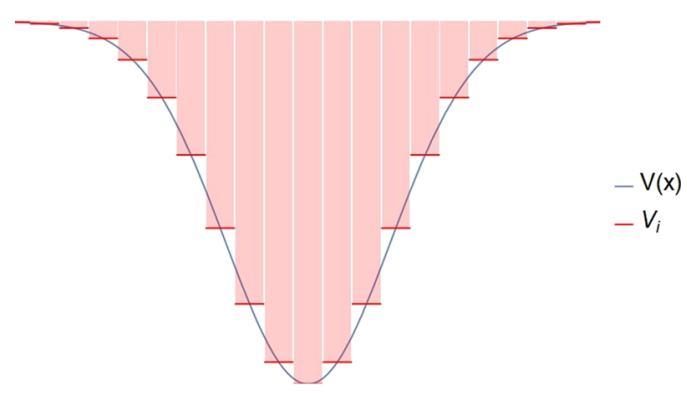

FIG. 7. A continuous potential $V(x)$ with a suitable discretization $V_{i}$.

necessarily unique inside the potential well. It is an open question of how such a degree of freedom affects the resulting expansion.

\section{ACKNOWLEDGMENTS}

The authors are thankful for support from the Department of mathematics and statistics at the Arctic University of Norway, from the Arizona Center for Mathematical Sciences at the University of Arizona, and for the support from the Air Force Office for Scientific Research under Grant No. FA9550-16-1-0088. Miro Kolesik was supported by the Air Force Office for Scientific Research under Grant No. FA9550-18-1-0183.

\section{APPENDIX: EVALUATION OF $b_{p}^{0}$}

The integral in (24) is computed as

$$
\begin{aligned}
& \pi^{-\frac{1}{2}}\left(\frac{3 \pi}{2}\right)^{-\frac{1}{6}} p^{-\frac{1}{6}} \int_{-d}^{d} d x f(x)\left\{a_{3} \cos \left(g_{1}(p)+x g_{2}(p)+\frac{\pi}{4}\right)+a_{2} \sin \left(g_{1}(p)+x g_{2}(p)+\frac{\pi}{4}\right)\right\} \\
& =\pi^{-\frac{1}{2}}\left(\frac{3 \pi}{2}\right)^{-\frac{1}{6}} p^{-\frac{1}{6}} \frac{1}{g_{2}(p)} \int_{g_{1}(p)-d g_{2}(p)+\frac{\pi}{4}}^{g_{1}(p)+d g_{2}(p)+\frac{\pi}{4}}\left[a_{2} \sin (y)+a_{3} \cos (y)\right] \mathrm{d} y \\
& =\left.\pi^{-\frac{1}{2}}\left(\frac{3 \pi}{2}\right)^{-\frac{1}{6}} p^{-\frac{1}{6}} \frac{1}{g_{2}(p)}\left[a_{3} \sin (y)-a_{2} \cos (y)\right]\right|_{y=g_{1}(p)-d g_{2}(p)+\frac{\pi}{4}} ^{y=g_{1}(p)+d g_{2}(p)+\frac{\pi}{4}} \\
& =\pi^{-\frac{1}{2}}\left(\frac{3 \pi}{2}\right)^{-\frac{1}{6}} p^{-\frac{1}{6}} \frac{1}{g_{2}(p)}\left[a_{3} \sin \left(g_{1}(p)+d g_{2}(p)+\frac{\pi}{4}\right)-a_{2} \cos \left(g_{1}(p)+d g_{2}(p)+\frac{\pi}{4}\right)\right. \\
& \left.-a_{3} \sin \left(g_{1}(p)-d g_{2}(p)+\frac{\pi}{4}\right)+a_{2} \cos \left(g_{1}(p)-d g_{2}(p)+\frac{\pi}{4}\right)\right] \\
& =\pi^{-\frac{1}{2}}\left(\frac{3 \pi}{2}\right)^{-\frac{1}{6}} p^{-\frac{1}{6}} \frac{2 \sin \left(d g_{2}(p)\right)}{g_{2}(p)}\left[a_{3} \cos \left(g_{1}(p)+\frac{\pi}{4}\right)+a_{2} \sin \left(g_{1}(p)+\frac{\pi}{4}\right)\right] .
\end{aligned}
$$

Using (A1) and the asymptotic forms of the Airy functions, the coefficients $b_{p}$ in (24) become

$$
\begin{aligned}
b_{p}^{0} \approx & \pi^{-\frac{1}{2}}\left(\frac{3 \pi}{2}\right)^{-\frac{1}{6}} p^{-\frac{1}{6}}\left[a_{2} \sin \left(g_{1}(p)+\frac{\pi}{4}\right)+a_{3} \cos \left(g_{1}(p)+\frac{\pi}{4}\right)\right] \\
& \pi^{-\frac{1}{2}}\left(\frac{3 \pi}{2}\right)^{-\frac{1}{6}} p^{-\frac{1}{6}} \frac{2 \sin \left(a g_{2}(p)\right)}{g_{2}(p)}\left[a_{3} \cos \left(g_{1}(p)+\frac{\pi}{4}\right)+a_{2} \sin \left(g_{1}(p)+\frac{\pi}{4}\right)\right] \\
= & \pi^{-1}\left(\frac{3 \pi}{2}\right)^{-\frac{1}{3}} p^{-\frac{1}{3}} \frac{2 \sin \left(a g_{2}(p)\right)}{g_{2}(p)}\left[a_{2} \sin \left(g_{1}(p)+\frac{\pi}{4}\right)+a_{3} \cos \left(g_{1}(p)+\frac{\pi}{4}\right)\right]^{2} .
\end{aligned}
$$


We take a further look at the trigonometric terms. After writing them in exponential forms, we find that one of the two exponentials can be neglected in the limit $p \gg 1$. Therefore we get

$$
\begin{aligned}
& \sin \left(g_{1}(p)+\frac{\pi}{4}\right) \approx \frac{1}{2 i}(-1)^{p} p^{\frac{1}{3}}\left(\pi^{2} c_{1}\right)^{-\frac{1}{2}}\left|\sin \left(c_{3} p^{\frac{1}{3}}\right)\right|^{-\frac{1}{2}} e^{i\left(\mu^{\frac{1}{2}} p^{\frac{1}{3}} \gamma V_{0}-\frac{1}{2} h(p)+\frac{\pi}{4}\right)}, \\
& \cos \left(g_{1}(p)+\frac{\pi}{4}\right) \approx \frac{1}{2}(-1)^{p} p^{\frac{1}{3}}\left(\pi^{2} c_{1}\right)^{-\frac{1}{2}}\left|\sin \left(c_{3} p^{\frac{1}{3}}\right)\right|^{-\frac{1}{2}} e^{i\left(\mu^{\frac{1}{2}} p^{\frac{1}{3}} \gamma V_{0}-\frac{1}{2} h(p)+\frac{\pi}{4}\right)} .
\end{aligned}
$$

Using (A3), we rewrite (A2) as

$$
\begin{aligned}
b_{p}^{0} \approx & \pi^{-1}\left(\frac{3 \pi}{2}\right)^{-\frac{1}{3}} p^{-\frac{1}{3}} \frac{2 \sin \left(d(3 \pi \varepsilon p)^{\frac{1}{3}}\right)}{g_{2}(p)}\left[a_{2} \frac{1}{2 i}(-1)^{p} p^{\frac{1}{3}}\left(\pi^{2} c_{1}\right)^{-\frac{1}{2}}\left|\sin \left(c_{3} p^{\frac{1}{3}}\right)\right|^{-\frac{1}{2}}\right. \\
& e^{i\left(\mu^{\frac{1}{2}} p^{\frac{1}{3}} \gamma V_{0}-\frac{1}{2} h(p)+\frac{\pi}{4}\right)} \\
& \left.+a_{3} \frac{1}{2}(-1)^{p} p^{\frac{1}{3}}\left(\pi^{2} c_{1}\right)^{-\frac{1}{2}}\left|\sin \left(c_{3} p^{\frac{1}{3}}\right)\right|^{-\frac{1}{2}} e^{i\left(\mu^{\frac{1}{2}} p^{\frac{1}{3}} \gamma V_{0}-\frac{1}{2} h(p)+\frac{\pi}{4}\right)}\right]^{2} \\
& =\pi^{-1}\left(\frac{3 \pi}{2}\right)^{-\frac{1}{3}} p^{-\frac{1}{3}} \frac{2 \sin \left(d(3 \pi \varepsilon p)^{\frac{1}{3}}\right)}{\mu^{\frac{1}{2}} p^{\frac{1}{3}} \gamma \varepsilon} \\
& \left(\frac{1}{2}(-1)^{p} p^{\frac{1}{3}}\left(\pi^{2} c_{1}\right)^{-\frac{1}{2}}\left|\sin \left(c_{3} p^{\frac{1}{3}}\right)\right|^{-\frac{1}{2}} e^{i\left(\mu^{\frac{1}{2}} p^{\frac{1}{3}} \gamma V_{0}-\frac{1}{2} h(p)+\frac{\pi}{4}\right)}\right)^{2}\left(\frac{a_{2}}{i}+a_{3}\right)^{2} \\
& =\left(\frac{3 \pi}{2}\right)^{-\frac{1}{3}} p^{-\frac{1}{3}} \frac{\sin \left(d(3 \pi \varepsilon p)^{\frac{1}{3}}\right)}{2(3 \pi \varepsilon p)^{\frac{1}{3}}} p^{\frac{2}{3}}\left(\pi^{3} c_{1}\right)^{-1}\left|\sin \left(2 d(3 \pi \varepsilon p)^{\frac{1}{3}}\right)\right|^{-1} \\
& e^{i 2\left(\mu^{\frac{1}{2}} p^{\frac{1}{3}} \gamma V_{0}-\frac{1}{2} h(p)+\frac{\pi}{4}\right)}\left(\frac{a_{2}}{i}+a_{3}\right)^{2} \\
& =\left(\frac{3 \pi}{2}\right)^{-\frac{1}{3}} \frac{\sin \left(d(3 \pi \varepsilon p)^{\frac{1}{3}}\right)}{2(3 \pi \varepsilon)^{\frac{1}{3}}}\left(\pi^{3} c_{1}\right)^{-1}\left|\sin \left(2 d(3 \pi \varepsilon p)^{\frac{1}{3}}\right)\right|^{-1} \\
& e^{i 2\left(\mu^{\frac{1}{2}} p^{\frac{1}{3}} \gamma V_{0}-\frac{1}{2} h(p)+\frac{\pi}{4}\right)}\left(\frac{a_{2}}{i}+a_{3}\right)^{2} .
\end{aligned}
$$

Thus

$$
\begin{aligned}
\left|b_{p}^{0}\right| & \approx \frac{\alpha}{V_{0}}\left|\frac{\sin \left(d(3 \pi \varepsilon p)^{\frac{1}{3}}\right)}{\sin \left(2 d(3 \pi \varepsilon p)^{\frac{1}{3}}\right)}\right| \\
& =\frac{\alpha}{V_{0}\left|\cos \left(d(3 \pi \varepsilon p)^{\frac{1}{3}}\right)\right|},
\end{aligned}
$$

where

$$
\alpha=\frac{\varepsilon^{\frac{1}{3}}}{2^{\frac{5}{3}} \pi}\left|\left(a_{3}-i a_{2}\right)\right|^{2} .
$$

${ }^{1}$ G. A. Gamow, “Zur quantentheorie des atomkernes,” Z. Phys. 51, 204-212 (1928).

${ }^{2}$ G. A. Gamow, "Zur quantentheorie des atomzertrummerung," Z. Phys. 52, 510-515 (1928)

${ }^{3}$ R. W. Gurney and E. U. Condon, "Quantum mechanics and radioactive disintegration," Phys. Rev. 33, 127-140 (1929).

${ }^{4}$ R. W. Gurney and E. U. Condon, "Quantum mechanics and radioactive disintegration," Nature 122, 439 (1928).

${ }^{5}$ J. J. Thomson, "On electrical oscillations and the effects produces by the motion of an electric sphere," Proc. London Math. Soc. s1-15, 197-218 (1884).

${ }^{6}$ J. A. J. F. Siegert, "On the derivation of the dispersion formula for nuclear reactions," Phys. Rev. 56, 750-752 (1939).

${ }^{7}$ R. E. Peierls, "Complex eigenvalues in scattering theory," Proc. R. Soc. London 253, 16-36 (1959).

${ }^{8}$ K. J. Le Couteur, "The structure of a non-relativistic s-matrix," Proc. R. Soc. London 256, 115-127 (1960).

${ }^{9}$ J. Humblet, "Theory of nuclear reactions," Nucl. Phys. 26, 529-578 (1961).

${ }^{10}$ T. Goto, "On the unstable states in quantum field theory," Prog. Theor. Phys. 21(1), 1-17 (1959).

${ }^{11}$ P. Lin, "Completeness relations and the resonant state expansions," Phys. Rev. C 47, 1903 (1993). 
${ }^{12}$ G. Garcia-Calderon, “An expansion of continuum wave functions in terms of resonant states,” Nucl. Phys. A 261, 130 (1976).

${ }^{13}$ V. N. Ostrovsky, O. I. Tolstikhin, and H. Nakamura, "Siegert pseudo state formulation of scattering theory: One-channel case," Phys. Rev. A 58, 2077 (1998).

${ }^{14}$ O. I. Tolstikhin, "Siegert-state expansion for nonstationary systems: Coupled equations in the one-channel case," Phys. Rev. A 73, 062705 (2006).

${ }^{15}$ O. I. Tolstikhin, "Siegert-state expansion for nonstationary systems. IV. Three-dimensional case,” Phys. Rev. A 77, 032712 (2008).

${ }^{16}$ T. Berggren, "On the use of resonant states in eigenfunction expansions of scattering and reaction amplitudes," Nucl. Phys. A 109, 265-287 (1968).

${ }^{17}$ R. M. More, "Theory of decaying states," Phys. Rev. A 4(5), 1782-1790 (1971).

${ }^{18}$ G. Garcia-Calderon and R. Peierls, "Resonant states and their uses," Nucl. Phys. A 265, 443-460 (1976).

${ }^{19}$ T. Bergren and P. Lin, "Resonant state expansion of the resolvent," Phys. Rev. C 47, 768 (1993).

${ }^{20}$ J. von Neumann, The Mathematical Foundations of Quantum Mechanics (Princeton University Press, 1932).

${ }^{21}$ P. A. M. Dirac, The Principles of Quantum Mechanics (Oxford at the Clarendon Press, 1930).

${ }^{22}$ I. M. Gelfand and N. Y. Vilenkin, Generalized Functions (Academic Press, 1964), Vol. 4.

${ }^{23}$ D. C. Brody, "Boirthogonal quantum mechanics," J. Phys. A: Math. Theor. 47, 035305 (2013).

${ }^{24}$ N. Moiseyev, Non-Hermitian Quantum Mechanics (Cambridge University Press, 2011).

${ }^{25}$ G. Garcia-Calderon, A. Mattar, and J. Villavicencio, "Hermitian and non-Hermitian formulations of the time evolution of quantum decay," Phys. Scr. T151, 014076 (2012).

${ }^{26}$ N. H. K. Sasada and G. Ordonez, "Resonant spectrum analysis of the conductance of an open quantum system and three types of fano parameter," Jpn. Phys. Soc. 80, 104707 (2011).

${ }^{27}$ Gonzalo Ordonez and N. Hatano, "The arrow of time in quantum systems and dynamical breaking of the resonance-antiresonance symmetry," J. Phys. A: Math. Theor. 50, 405304 (2017).

${ }^{28} \mathrm{~N}$. Hatano and G. Ordonez, "Time-reversal symmetric resolution of unit without background integrals in open quantum systems," J. Math. Phys. 55, 122106 (2014).

${ }^{29}$ N. Hatano, "Probabilistic interpretation of resonant states," Pramana 73, 553 (2009).

${ }^{30}$ J. Rosenkrantz de Lasson, P. T. Kristensen, J. Mørk, and N. Gregersen, "Roundtrip matrix method for calculating the leaky resonant modes of open nanophotonic structures," J. Opt. Soc. Am. A 31(10), 2142-2151 (2014).

${ }^{31}$ P. T. Kristensen and S. Hughes, "Modes and mode volumes of leaky optical cavities and plasmonic nanoresonators," ACS Photonics 1(1), 2-10 (2014).

${ }^{32}$ E. F. Franchimon, K. R. Hiremath, R. Stoffer, and M. Hammer, "Interaction of whispering gallery modes in integrated optical microring or microdisk circuits: Hybrid coupled mode theory model," J. Opt. Soc. Am. B 30(4), 1048-1057 (2013)

${ }^{33}$ A. Settimi, S. Severini, and B. J. Hoenders, "Quasi-normal-modes description of transmission properties for photonic bandgap structures,” J. Opt. Soc. Am. B 26(4), 876-891 (2009).

${ }^{34}$ R.-C. Ge, J. F. Young, and S. Hughes, "Quasi-normal mode approach to the local-field problem in quantum optics,” Optica 2(3), 246-249 (2015).

${ }^{35}$ F. Yang, H. Liu, H. Jia, and Y. Zhong, "Analytical description of quasi-normal mode in resonant plasmonic nano cavities," J. Opt. 18(3), 035003 (2016).

${ }^{36}$ O. I. Tolstikhin, V. N. Ostrovsky, and H. Nakamura, "Siegert pseudo-states as a universal tool: Resonances, $S$ matrix, green function," Phys. Rev. Lett. 79, 2026-2029 (1997).

${ }^{37}$ Resonances the Unifying Route Towards the Formulation of Dynamical Processes Foundations and Applications in Nuclear, Atomic and Molecular Physics, Lecture Notes in Physics, edited by E. Brändas and N. Elander (Erkki Brändas, 1987).

${ }^{38}$ R. de la Madrid, G. García-Calderón, and J. Gonzalo Muga, "Resonant expansions in quantum mechanics," Czech. J. Phys. $\mathbf{5 5}, 1141$ (2005).

${ }^{39}$ N. Moiseyev, "Quantum theory of resonances: Calculating energies, widths and cross-sections by complex scaling," Phys. Rep. 302(5-6), 212-293 (1998).

${ }^{40}$ A. Teleki, P. Jakobsen, J. V. Moloney, M. Kolesik, J. M. Brown, and E. M. Wright, "Metastable electronic states and nonlinear response for high-intensity optical pulses," Optica 1(5), 323 (2014).

${ }^{41}$ A. Bahl, J. K. Wahlstrand, S. Zahedpour, H. M. Milchberg, and M. Kolesik, "Nonlinear optical polarization response and plasma generation in noble gases: Comparison of metastable-electronic-state-approach models to experiments," Phys. Rev. A 96, 043867 (2017).

${ }^{42}$ A. Couairon, E. Brambilla, T. Corti, D. Majus, O. J. Ramirez-Gongora, and M. Kolesik, "Practitioner's guide to laser pulse propagation models and simulation,” Eur. Phys. J.: Spec. Top. 199(1), 5-76 (2011).

${ }^{43}$ E. Lorin, S. Chelkowski, and A. Bandrauk, "Maxwell-Schrödinger-plasma (MASP) model for laser-molecule interactions: Towards an understanding of filamentation with intense ultrashort pulses," Physica D 241(12), 1059-1071 (2012).

${ }^{44}$ H. L. Cycon, R. G. Froese, W. Kirsch, and B. Simon, Schrödinger Operators (Springer-Verlag, 1987).

${ }^{45}$ J. M. Brown, P. Jakobsen, A. Bahl, J. V. Moloney, and M. Kolesik, "On the convergence of quantum resonant-state expansion," J. Math. Phys. 57, 032105 (2016).

${ }^{46}$ W. Elberfeld and M. Kleber, "Tunneling from an ultrathin quantum well in a strong electrostatic field: A comparison of different methods," Z. Phys. B: Condens. Matter 73(1), 23-32 (1988).

${ }^{47}$ S. Geltman, "Ionisation dynamics of a model atom in an electrostatic field," J. Phys. B: At. Mol. Phys. 11(19), 3323-3337 (1978).

${ }^{48}$ A. Teleki, E. M. Wright, and M. Kolesik, "Microscopic model for the higher-order nonlinearity in optical filaments," Phys. Rev. A 82, 065801 (2010).

${ }^{49}$ A. Emmanouilidou, "Stark and field-born resonances of an open square well in a static external electric field," J. Chem. Phys. 122, 194101 (2005).

${ }^{50}$ B. Gyarmati and T. Vertse, "On the normalization of gamow functions," Nucl. Phys. A 160, 523-528 (1971). 
${ }^{51}$ P. L. Kapur and R. Peierls, "The dispersion formula for nuclear reactions,” Proc. R. Soc. London 166, 277-295 (1939).

${ }^{52}$ N. Dunford and J. T. Schwartz, Linear Operators. Part II. Spectral Theory (Wiley, 1963), Theorem 3 p. 1192.

${ }^{53}$ I. W. Herbst, "Unitary equivalence of Stark Hamiltonians," Math. Z. 155, 55-70 (1977).

54 J. E. Avron and I. W. Herbst, "Spectral and scattering theory of Schrodinger operators related to the Stark effect," Commun. Math. Phys. 52, 239-254 (1977).

${ }^{55}$ B. Simon, "Phase space analysis of simple scattering systems: Extensions of some work of Enss," Duke Math. J. 46(1), 119-168 (1979).

${ }^{56}$ O. Valee and M. Soares, Airy Functions and Applications to Physics (Imperial College Press, 2004).

${ }^{57}$ NIST Digital Library of Mathematical Functions, http://dlmf.nist.gov/, Release 1.0.18 of March 3, 27, edited by F. W. J. Olver, A. B. Olde Daalhuis, D. W. Lozier, B. I. Schneider, R. F. Boisvert, C. W. Clark, B. R. Miller, and B. V. Saunders. 